\title{
Vivre avec une ventilation mécanique au quotidien : ergonomie et sécurité au domicile et à l'extérieur
}

\author{
Perrine Delalande, Geert Wasteels
}

L'évolution de certaines pathologies neuromusculaires conduit parfois à la nécessité d'une ventilation mécanique. Si l'adaptation de la ventilation est généralement réalisée à l'hôpital, la poursuite du traitement à domicile dans de bonnes conditions nécessite un environnement matériel et humain adapté pour garantir la sécurité et la qualité de vie de la personne.

Outre le ventilateur, le choix et le juste réglage de l'interface et de l'appareil d'humidification contribuent au confort du patient et à la qualité du traitement. La fixation du matériel au fauteuil et au patient contribue à sa sécurité.

À domicile, le patient est accompagné par des aidants familiaux ou professionnels. Il convient de veiller à leur formation afin qu'ils puissent être à l'aise avec le matériel et pour que celui-ci complique le moins possible le quotidien, l'objectif étant que le patient garde la meilleure qualité de vie possible.

En sortie à l'extérieur, le risque potentiel de défaillance du matériel devrait inciter le patient à s'équiper d'un " kit de secours " pour se déplacer en sécurité.

\section{Le point de vue des patients}

Quelle adaptation au domicile en cas

de dépendance à la ventilation?

Il n'y a pas à proprement parler d'adaptation spécifique du domicile pour la ventilation. Mais alors de quoi une personne ventilée a-t-elle besoin dans sa maison pour assurer un traitement sûr et efficace? Pour le savoir, nous avons réalisé une enquête auprès des patients de l'hôpital d'Inkendaal en Belgique chez des personnes ventilées la nuit uniquement, ou de jour et de nuit. Les questions qui leur étaient posées relèvent d'une interrogation centrale : " outre votre respirateur, de quelles aides techniques et appareillages avez-vous besoin en qualité de patient ventilé lorsque vous êtes à l'intérieur de la maison?". Il n'est pas étonnant que leurs réponses tournent autour de la sécurité. Une première problématique porte sur le bon positionnement de l'interface (embout buccal ou nasal) Une autre préoccupation récurrente concerne la réserve d'alimentation électrique du respirateur.

Un problème fréquent concerne la fixation de l'embout buccal ou nasal et des tuyaux du circuit respiratoire au lit (Figures 1a, 1b) comme sur le fauteuil roulant (Figure 1c). Pour répondre à cette préoccupation, des crochets pour fixer les tuyaux ont été réalisés grâce à une imprimante 3D. De même, un support (collier) placé sur les épaules de la personne ventilée vient fixer l'embout buccal devant sa bouche, même si elle bouge dans son fauteuil (Figure 2).

Qu'en est-il de la ventilation en dehors

du domicile?

C'est la même histoire : la sécurité avant tout!

Les pannes mécaniques font partie des problèmes

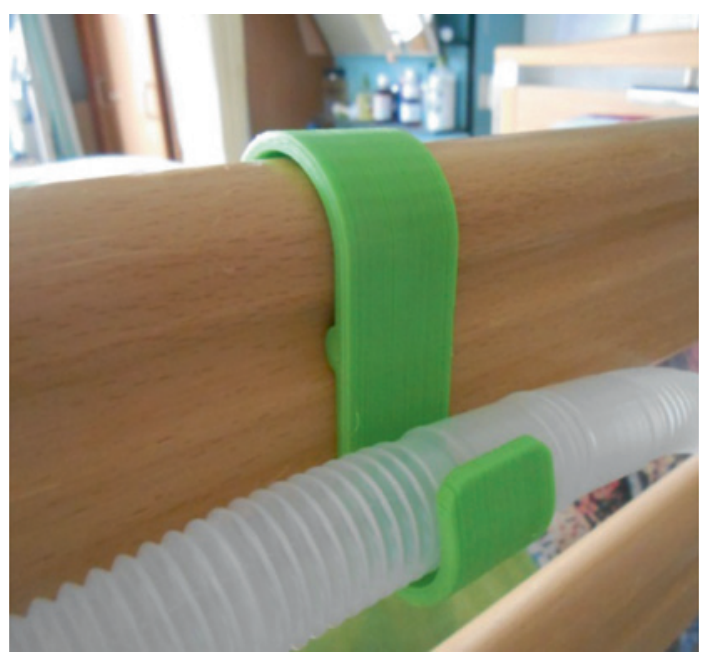

Figure 1a

Fixation du tuyau au bord du lit. 


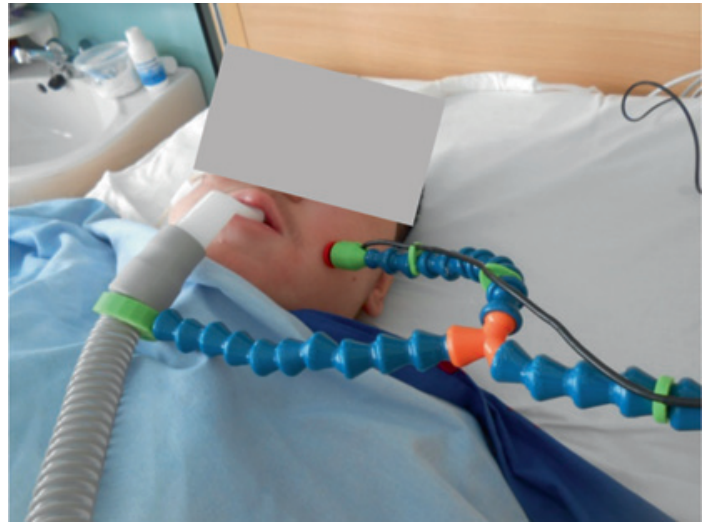

Figure $1 \mathrm{~b}$

Support maintenant l'embout buccal au lit (combiné avec un système d'appel).

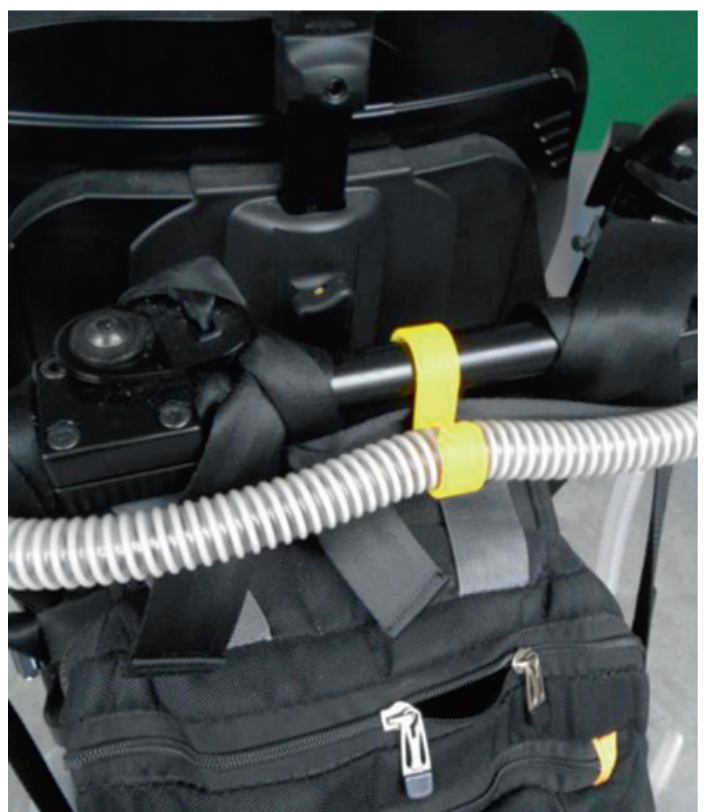

Figure 1c

Système de fixation au fauteuil roulant.

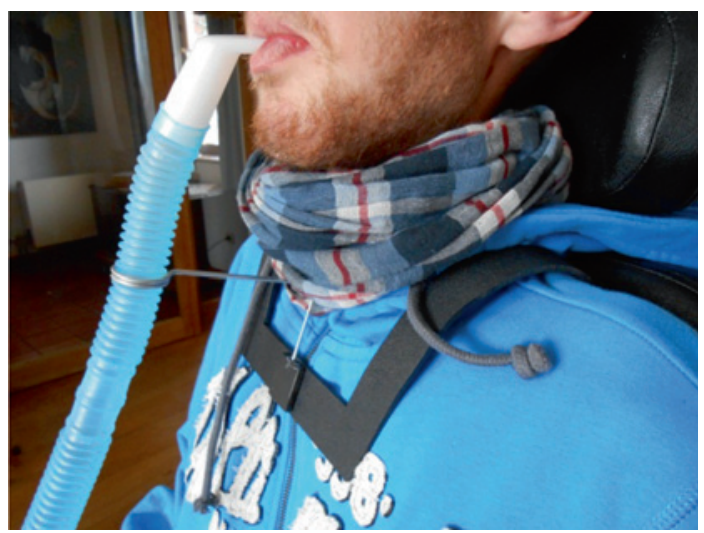

Figure 2

Support maintenant l'embout buccal. les plus redoutés, qu'elles soient mécaniques ou liées à la réserve d'alimentation électrique du respirateur. Pour limiter ces risques, le patient a intérêt à avoir avec lui des pièces de réserve ou rechange (une interface, un circuit ou du ruban adhésif...) et, peut-être le plus important, un ballon de réanimation (BAVU). Nous leur conseillons également de disposer d'un câble de charge et une batterie externe supplémentaire pour le ventilateur.

Comme au domicile, l'accent est mis en extérieur sur la fixation des tuyaux et le positionnement de l'embout buccal ou nasal. La continuité de la ventilation mécanique doit être considérée comme la première priorité.

Elle est assurée notamment par des adaptations (crochets, etc.) et des aides techniques, réalisés avec une imprimante 3D.

\section{Matériel nécessaire à domicile : prescripteur et prestataire ont un rôle majeur}

\section{Les ventilateurs}

\section{Des progrès incontestables}

Aujourd'hui, les machines sont moins encombrantes et moins lourdes. Elles sont presque toutes équipées de sacoches de transports. Les batteries ont une plus grande durée d'autonomie. Les écrans sont plus informatifs, indiquant notamment le niveau de charge des batteries et même, pour certains, le temps estimé restant d'autonomie.

Ces éléments ont permis aux patients de gagner en liberté de mobilité.

La possibilité d'enregistrer plusieurs programmes permet par exemple d'enregistrer un "programme jour " et un "programme nuit " sur chacun des ventilateurs, et éventuellement d'autres adaptés à des situations de la vie quotidienne, ou selon le besoin de gonfler/dégonfler le ballonnet. Certains ventilateurs permettent même de nommer ces différents programmes pour une meilleure information. Ces différents réglages "pré-programmés" permettent notamment d'utiliser le deuxième ventilateur en "machine de secours" en cas de panne. Les données de ventilation gardées en mémoire permettent au prescripteur (outre le suivi de la qualité de la ventilation), d'affiner le réglage des seuils d'alarmes pour sécuriser et limiter les alarmes intempestives. Le patient et son entourage gagnent en confort et en sécurité.

Alarmes : pouvoir les choisir, les inhiber, bien les régler et former les aidants

Les alarmes sont primordiales pour la sécurité du patient, encore faut-il trouver " la juste alarme " pour que la sécurité soit optimisée. 
L'interprétation des messages peut s'avérer problématique. Lorsqu'une alarme sonne, les messages qui apparaissent à l'écran ne sont pas toujours interprétables par l'environnement du patient et il n'est pas facile de trouver rapidement l'information appropriée dans les notices, surtout "dans l'urgence". Les manuels ne sont pas "pratiques". Sur certains ventilateurs, il existe un tutoriel intégré qui indique ce qui se passe, et comment régler le problème. Sur ce point, la ventilation "à domicile" implique une bonne formation des aidants pour les rassurer et sécuriser le patient.

Les alarmes posent également un risque de pollution sonore. Leur mauvais réglage et leur déclenchement répété, a fortiori la nuit, peut altérer la qualité de vie des patients. Par ailleurs, les alarmes intempestives peuvent conduire le patient et son accompagnant à banaliser et donc négliger une alarme qui peut, en réalité, signaler un risque vital.

Malheureusement, sur la plupart des ventilateurs le patient n'a pas accès à l'écran, qui se trouve dans son dos... Il doit toujours solliciter un tiers pour visualiser les informations (message d'alarme, niveau de charge...).

\section{La mise en charge}

Le système de mise en charge doit être simple et pratique. a défaut, il existe un risque de mauvais branchement ou de débranchement dans la sacoche. Des batteries qui ne sont pas correctement chargées font perdre au patient son autonomie de déplacement en sécurité.

Là encore, il convient de former l'entourage à vérifier le témoin de charge en façade du ventilateur.

\section{L'installation sur le fauteuil}

Tout le matériel du patient (respirateur, "sac d'urgence ", commande tierce personne, effets personnels, parfois matériel d'alimentation entérale, etc.) doit pouvoir être positionné sur le fauteuil roulant électrique, et correctement pour ne pas se détériorer. Les sacoches ne peuvent pas toujours être fixées comme le fabricant l'a prévu et l'accessibilité aux informations ou aux prises est parfois difficile.

Le patient doit pouvoir bouger son dossier pour soulager ses appuis, ce qui peut modifier la position d'un embout buccal. Des tuyaux peuvent se coincer ou des câbles se déconnecter... Ce point mérite d'être pris en compte dans le choix d'un ventilateur ou d'un nouveau fauteuil. Afin d'optimiser l'ergonomie, il y aurait un intérêt au travail conjoint entre fabricants de ventilateurs et fabricants de fauteuils en particulier pour l'intégration du matériel (Figure 3).

\section{L'interface}

Le choix d'une interface est guidé d'une part par l'efficacité du traitement, d'autre part par la

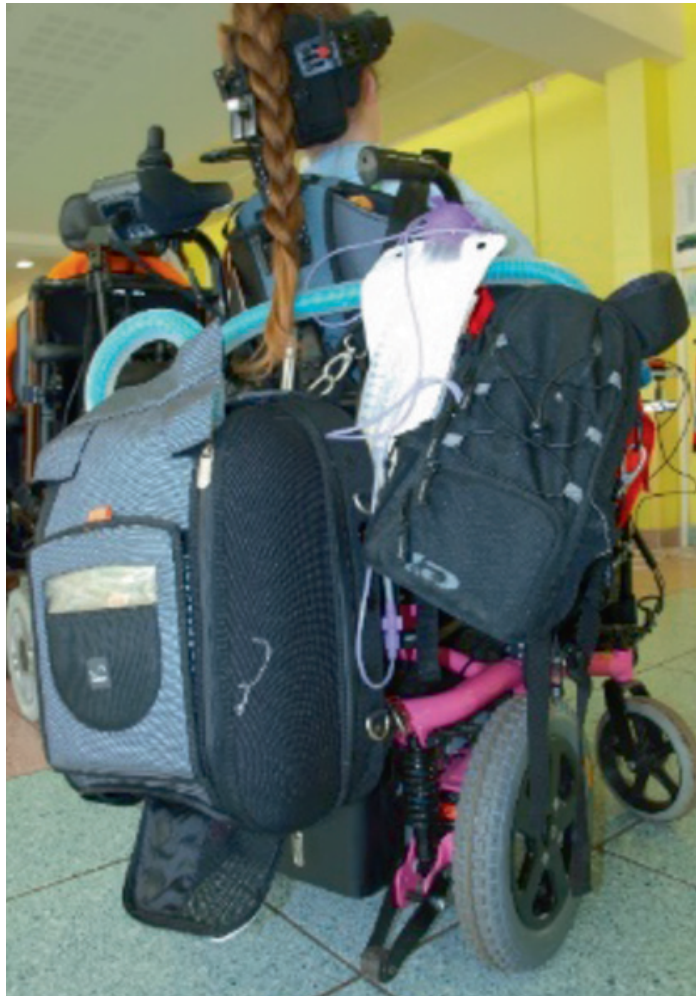

Figure 3

L'installation du matériel de ventilation sur le fauteuil roulant est parfois complexe.

tolérance du patient qui reste un paramètre majeur d'observance.

En ventilation "non invasive ", il est assez classique que le patient ait à sa disposition plusieurs interfaces qu'il choisit en fonction de son état clinique et de son confort.

- Embout buccal : il en existe différentes formes, coudées ou droites, surface de l'ouverture, plus ou moins souples... Le ressenti du patient prime et son acceptation dépend du choix du bon matériel.

- Masque nasal ou narinaire : une multitude de masques différents existe. Ils sont de plus en plus confortables. Cependant le risque de lésion cutanée par hyper-appui incite à choisir et laisser plusieurs interfaces à disposition du patient pour qu'il puisse alterner. Par ailleurs, pour un patient ventilé au masque en continu, l'utilisation d'un embout buccal peut être ponctuelle et adaptée pour la réalisation de soins d'hygiène et de confort au niveau du visage et du cuir chevelu, ou en cas d'épistaxis.

Pour les patients trachéotomisés, il existe aussi une large gamme de canules très différentes. Au-delà de la charrière, elles sont toutes caractérisées par un diamètre interne et externe, un rayon de courbure, une souplesse, une longueur, la possibilité ou non d'y associer une chemise interne, la forme de la 
collerette... Autant de critères auxquels chaque patient sera sensible, en fonction de son anatomie notamment.

\section{L'humidification}

Les nouvelles recommandations sont d'utiliser pour les patients trachéotomisés un circuit chauffant au moins sur la branche inspiratoire. Dans le cas d'une ventilation avec un circuit double branche, cela peut provoquer une condensation importante dans la branche expiratoire, source de risque: inhalation d'eau, inondation de la valve expiratoire et défaillance du ventilateur. Cela impose la formation et la vigilance du patient et de ses aidants à la surveillance et à l'adaptation du réglage.

L'utilisation d'un circuit chauffé sur les deux branches permet de réduire ce risque.

\section{Les sorties à l'extérieur du domicile}

La contrainte principale est d'avoir à disposition le matériel nécessaire en cas de panne ou de problème technique

Pour cette raison, nous conseillons aux patients ventilo-dépendants de sortir équipés d'un deuxième ventilateur "de secours", d'un circuit de rechange (ou d'un rouleau de scotch !), d'une deuxième interface et d'un BAVU. Idéalement, l'ensemble de ce matériel est placé dans un sac dédié et accessible. Les coordonnées du prestataire sont généralement indiquées sur le matériel. Avant de sortir, il faut vérifier que le niveau de charge des batteries est suffisant pour couvrir la durée du déplacement et avoir un câble de charge sur soi en cas d'imprévu.

Certains patients ont aménagé et équipé leur véhicule personnel pour accueillir tout ce matériel, ainsi que le nécessaire de désencombrement si besoin (aspiration endo-trachéale ou aide à la toux). Pour éviter d'utiliser les batteries pendant le trajet, certains ont installé un transformateur afin de disposer d'une prise $220 \mathrm{~V}$. Des cordons allume-cigare sont également proposés pour certains ventilateurs.

Enfin, il est possible de modifier le volume sonore de l'alarme en fonction de l'environnement où l'on se trouve.

\section{Environnement humain : former}

\section{les aidants et leur établir une check-list}

Pour que la ventilation à domicile soit bien conduite et limite le moins possible l'autonomie dans la vie quotidienne, il est important que les aidants soient à l'aise avec le matériel et les grands principes du traitement. Il faut prévoir de les former notamment à la manipulation du ventilateur, à la mise en charge des batteries, ainsi qu'à l'interprétation des messages et aux actions à mener le cas échéant. Le traitement implique une multitude de petits gestes simples et quotidiens. La rédaction d'une "check-list" énumérant tous ces gestes est une bonne aide pour ne pas en oublier.

\section{Conclusion}

La ventilation mécanique est un traitement vital généralement initié à l'hôpital. Sa mise en œuvre à domicile, dans les conditions de vie, nécessite des adaptations qui sont guidées par trois objectifs clés : la qualité de vie, l'observance et la sécurité.

Le choix du ventilateur pour un patient dépend de la connaissance du matériel par le prescripteur et le prestataire pour le régler correctement. Mais il est guidé également par les habitudes de vie du patient (besoin en autonomie de batteries, nombre de programmes), la tolérance, les possibilités d'installation de l'appareil sur le fauteuil.

Au-delà du ventilateur, chaque accessoire doit être choisi, réglé et installé avec comme leitmotivs l'ergonomie et la sécurité. L'industrie propose déjà des choses intéressantes. D'autres méritent encore d'être développées, par exemple des crochets de fixation des circuits et un support pour embout buccal fixé au patient et non au fauteuil.

Enfin, l'environnement humain et la formation des aidants sont essentiels pour garantir la sécurité et la qualité de vie du patient.

Living with mechanical ventilation on a daily basis: ergonomics and safety at home and outside

\section{LIENS D'INTÉRÊT}

Les auteurs déclarent n'avoir aucun lien d'intérêt concernant les données publiées dans cet article.

\section{Retrouvez toutes les Actualités de la Myologie sur les sites de:}

la Société Française de Myologie www.sfmyologie.org

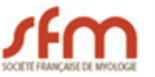
la filière de santé neuromusculaire FILNEMUS www.filnemus.fr

